NISTIR 7619

\title{
Visualization of Structural Behavior under Fire
}

\author{
Dilip Banerjee \\ John Gross \\ Materials and Construction Research Division \\ Building and Fire Research Laboratory \\ William Hess \\ Marc Olano \\ Judith Terrill \\ Mathematical and Computational Science Division \\ Information Technology Laboratory
}

August 2009

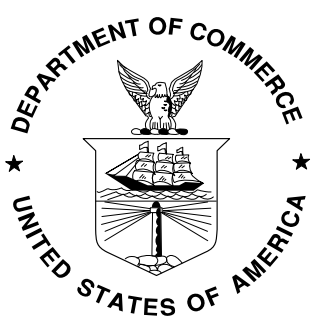

U.S. Department of Commerce

Gary Locke, Secretary

National Institute of Standards and Technology

Patrick D. Gallagher, Deputy Director 


\begin{abstract}
An integrated environment was created to study the interaction among fire, heat transfer, and structural deformation from a typical room fire. The fire, thermal, and structural data were linked with a separate 3-D visualization capability, to provide the ability to visualize in real time the thermal and structural behavior of a chosen structural component in a room subjected to a typical fire in an Immersive Visualization Environment (IVE).

In this initial study a single beam in a room was used for our structural system. A sequential process was followed in which first the Fire Dynamics Simulator (FDS) program was used to simulate the start and development of fire in the room. Then a second computer program was used to calculate how the gas temperature computed by the FDS program propagated into the beam. Finally, a third computer program was used to compute how the beam deformed over time due to combined effects of thermal and mechanical loads.

The three outputs from these computer programs were used in two separate visualization methods that were developed to display the computed results in an IVE. One visualization method was based on polygons and the other was a Graphics Processing Unit (GPU) based ray traced volumetric rendering.

In the next phase of the project, the behavior of other structural components including connections will be studied. Also, the capability to extract and dynamically plot relevant numerical data at specified surfaces or at a specific location will be incorporated. This capability might be useful in providing a real time insight into the possibility of an impending collapse due to a fire.
\end{abstract}




\section{Contents}

1. Introduction

2. Fire Dynamics

3. Heat Transfer Modeling

4. Structural Modeling

5. Data Transfer to the Visualization Facility

6. Immersive Visualization

6.1 Introduction

6.2 Polygon Based Visualization of Fire

6.3 GPU Based Ray Traced Volumetric Visualization of Fire

7. Discussion and Future Work

8. Acknowledgements

9. Disclaimer

10. Bibliography

11. List of Figures

A. Appendix

A.1 FDS Input File for Test Case 


\section{Introduction}

Analysis of structural systems under fire conditions involves the prediction of performance of the entire structural system at, or near, its limit state of collapse, since an uncontrolled fire is considered an extreme (though not improbable) event. Development of accurate models to predict complex structural system behavior resulting from the effects of thermal expansion and diminished mechanical properties at elevated temperatures requires the availability of robust computational models, validated against large-scale tests under real fire exposures.

Spatially and temporally varying numerical quantities such as temperature or stress within the continuum (volume within a compartment or structural element) can be described by color. Analysis results, e.g. gas temperatures, can be presented in terms of a color spectrum whereby each color represents a temperature range.

In order to numerically model the behavior of structures under fire, three separate and independent analyses are typically conducted to predict 1) gas temperatures in a compartment fire, 2) heating of structural elements exposed to hot gases from fire, and 3) structural responses due to both thermal and mechanical loads. Each of these analyses generates a vast amount of data at varying time and space intervals within the computational domain. There is a need to find out a way to integrate the data display in a visual context.

One such approach is to create an integrated environment to study the interaction among fire, heat transfer, and structural deformation resulting from a typical room fire. In order to achieve this goal, the analysis data sets need to be integrated with a visualization tool that can display vividly these results. The visualization tool should provide the ability to display the results in an immersive visualization environment (IVE).

The goal of this present study is to link the fire, thermal, and structural data with a separate 3-D visualization capability, which provides the ability to visualize the thermal 
and structural behavior of a chosen structural component in a room subjected to a typical fire in an IVE. This capability provides a high-quality visualization of the massive data generated from the simulation in a structured manner.

In this initial study, a single beam in a room is used as the structural system. Then a sequential process was followed in which first the Fire Dynamics Simulator (FDS) [1] program is used to simulate the start and propagation of fire in the room. Then a second computer program is used to calculate how the gas temperature computed by FDS heats the beam due to the thermal conduction. Finally, a third computer program is used to compute how the beam deforms over time due to the combined effects of thermal and mechanical loads. Two commercial finite element software packages (ABAQUS and ANSYS) are used to perform both the thermal and structural analyses. In this article, only the results from ABAQUS simulations are discussed as a test case [2].

\section{Motivation}

NIST's recommendation 9 as part of its investigation into the collapse of World Trade Center Towers [3] states that "NIST recommends the development of: (1) performancebased standards and code provisions, as an alternative to current prescriptive design methods, to enable the design and retrofit of structures to resist real building fire conditions, including their ability to achieve the performance objective of burnout without structural or local floor collapse and (2) the tools, guidelines, and test methods necessary to evaluate the fire performance of the structure as a whole system”.

Recommendation 9 further states that “....This performance-based capability should include, but not be limited to publicly available computational software to predict the effects of fires in tall buildings - developed, validated, and maintained through a national effort-for use in the design of fire protection systems and the analysis of building response to fires.”

Standards development organizations such as American Institute of Steel Construction have already begun developing performance-based provisions to consider the effects of fire in structural design. This preliminary study is an effort toward the development of a performance based system to evaluate the fire performance of the structure using fire as a design load. The building layout (compartmentation, geometry), windows (ventilation), materials of construction, passive and active fire protection systems, and amount and location of combustibles are included in the new approach.

The use of this approach requires a fire model that can predict the development and propagation of building fires, influenced by the building characteristics noted above, using the application of fire science. This approach enables sensitivity studies to be conducted to establish the most important factors affecting the fire performance of the entire structure, to understand the behavior of different types of structural systems exposed to the same fire, and to evaluate the effectiveness of alternate rehabilitation, design, and fire protection strategies. 
In case of fire in a building, the important aspects are fire propagation and interaction between the fire and the building structure. Realistic models describing fire initiation and growth are needed for such simulations.

\section{Fire Dynamics}

For the fire simulations in this study, the large-eddy simulation code Fire Dynamics Simulator (FDS) is used. FDS provides time resolved temperature, $\mathrm{CO}, \mathrm{CO}_{2}$, and soot distribution in the compartment. It has an accompanying visualization tool, Smokeview [4].

The FDS model for a typical room was built as shown in Figure 1. The room has 3 windows and an opening. The room is $16 \mathrm{~m}$ in length, $8 \mathrm{~m}$ in width, and $4 \mathrm{~m}$ in height. The walls of the room are made of typical gypsum board. The thermophysical properties of the gypsum were assumed to be independent of temperature.

By default, FDS assumes that the exterior boundary of the computational domain is a solid wall. An open vent was assumed at one end of the room to denote a passive opening to the outside. The fire was applied uniformly over the rectangular floor area of $14 \mathrm{~m}$ by $8 \mathrm{~m}$ using a ramped heat release rate function. This function was chosen such that fire developed fully after $1 \mathrm{~s}$ and began to decay after $529.5 \mathrm{~s}$ and was finally exhausted after $1059 \mathrm{~s}$. The heat release rate per unit area was assumed to be $1260 \mathrm{~kW} / \mathrm{m}^{2}$. A typical FDS input file is shown in Appendix A.

For the test case, a beam is positioned above the ceiling in the middle of the room along the width of the room as shown in the schematic in Figure 2. The beam is not part of the FDS simulation. For the fire/heat transfer interface, the specification of the bounding gas temperatures at all solid surfaces is required. Spatially and temporally varying timetemperature data need to be transferred from the fire model to the heat transfer model. The adiabatic surface temperature concept in FDS allows for the output of the fire model to serve as a boundary condition for the thermal model. The Adiabatic Surface Temperature (AST) was used to capture the time dependent temperature underneath the beam's bottom flange location as shown by the points in Figure 1.

The temperature of a perfect insulator exposed to the same heating condition as the real surface is referred to as the adiabatic surface temperature (AST) and by definition, the total net heat flux to this ideal surface is zero. ASTs are the temperatures that plate thermometers would measure if placed in the vicinity of surfaces of interest. The time history of the AST can be output at chosen nodes defined by spatial coordinates and orientation. Since the spatial resolution of fire models is typically much coarser that that of the heat transfer model, the AST concept is a useful approach to transfer the gas temperature data to the heat transfer model. Using an appropriate parameter in the FDS input file, these AST quantities were output at regular intervals at specified points (at 0.8 
$m$ increment along the width of the room) for use as a boundary condition to the heat transfer model. Refer to the FDS user manual for further detail [1].

\section{Heat Transfer Modeling:}

It is assumed that the elevated temperature generated in the beam due to fire effects has a strong influence on the mechanical behavior of the beam and that the mechanical behavior of the beam influences the thermal response weakly. Hence, a sequential thermal stress analysis is performed. In this study, the commercial finite element software, ABAQUS, was used for both the heat transfer and subsequent structural analyses. The thermal analysis model was run first and the results formed the input for the structural analysis model. The thermal analysis data (nodal temperatures) were output at specified time intervals for later use as input to the structural analysis model.

The heat transfer was modeled as a simple heat conduction problem as a test case. A W24X76 steel beam was chosen. The beam was assumed to have a half inch of fireproofing uniformly applied on it. Both solid brick elements and shell elements were used in separate analyses.

For the shell model, the beam was comprised of upper and lower flanges and the web as separate regions. Conventional shell with composite lay-up utility in ABAQUS was used. A 3-layer model consisting of the lower insulation layer, steel layer, and the upper insulation layer in sequence was used in the shell model definition. Section integration was used during analysis. A standard 4-node quadrilateral, heat transfer shell element (DS4) with linear geometric order was used to discretize the computational domain.

The heat transfer finite elements used here are intended to model heat transfer in shelltype structures. They provide the values of temperature at a number of points through the thickness at each shell node. The temperature output can be transferred directly to the equivalent stress analysis shell elements for sequentially coupled thermal-stress analysis. The temperature variation is assumed to be piecewise quadratic through the thickness.

In order to use the temperature results data that are generated during ABAQUS/Standard thermal analysis directly as input to a subsequent thermal-stress analysis, the mesh and the specification of the number of temperature points in the shell sections must be identical in both the heat transfer and the stress analysis models. Additionally, multilayered heat transfer shell elements should have the same number of integration points in each layer.

In the 3-D model, the steel and the fireproofing materials were modeled as separate domains. Temperature independent thermal properties were assumed for both of these materials. An 8-node heat transfer brick element (DC3D8) with linear geometric order was used. The beam was initially assumed to be at ambient temperature. The time dependent temperature data (AST) computed by the FDS model was applied at specific 
locations in the bottom flange as boundary conditions. A snapshot of the temperature data at 1666 seconds into the thermal computation is shown in Figure 3. The heat transfer calculation was continued for 5000 seconds. The nodal temperature data were stored at a user-chosen time interval throughout the simulation. These data are used during thermal stress calculations later on as discussed in the following section.

In this study, results obtained using 3-D solid elements are shown as a test case. It may be mentioned here that a properly refined finite element mesh comprising of shell elements can produce solutions comparable to those generated by a similarly refined mesh of 3D solid elements [5].

\section{Structural Modeling:}

The structural modeling comprises static analysis over two separate analysis steps using ABAQUS/Standard module. In the first step, a gravity load is applied. The finite element model developed for thermal analysis is copied to make a structural analysis model and the thermal elements are changed to structural elements. The new model has all the necessary thermophysical and mechanical properties specified during the heat transfer analysis. The restart capability in ABAQUS is used during structural analysis.

In the second step, the finite element model developed in the first step is copied into a new model. The mechanical load and boundary conditions applied in the first step are readily available in this new model. In this step, the thermal load is applied as computed by the heat transfer program described in section 4 . This is done by reading the corresponding ABAQUS output database (results file) from the heat transfer analysis. Then a restart analysis is performed in step 2. ABAQUS allows for automatic interpolation of data between the thermal analysis mesh and the structural analysis mesh over the same geometry.

In the first step, a uniform pressure load of $4.2 \mathrm{kPa}$ (kilopascal) (equal to the sum of dead and live loads) was applied on the top flange of the beam. A standard, linear 8-node hexahedral element (C3D8I) was used. At either end of the beam in the mid point of the web, fixed boundary conditions were used (both displacement and rotations are zero for all directions). A general static analysis was first performed over $10 \mathrm{~s}$. ABAQUS/Implicit direct solver employing Full Newton solution technique was used. The time step was allowed to vary based on the rate of solution convergence.

In the second step, the thermal load computed in the heat transfer analysis (as discussed in section 4 above) was applied over the entire domain in the new model. The thermal data from the heat transfer analysis was read over the entire domain for the entire time period in thermal analysis. The nonlinear geometry option was turned on for this structural analysis. The structural analysis was used for the entire duration of the fire. A typical contour of the deformation in the y-direction with a scaling (magnification) factor of 10 is shown in Figure 4. Here, the color blue represents maximum vertical 
displacement and the color red represents minimum vertical displacement. The maximum vertical displacement was obtained in the middle region as expected.

The simulation results were checked to ensure that there was no instability. In particular, no negative eigenvalue was obtained in the simulation. Negative eigenvalues indicate that the load to cause the structure to buckle is of the opposite sign to the applied load.

\section{Data Transfer to the Visualization Facility:}

ABAQUS output database (Job-name.odb) is basically a neutral file used to store analysis results. The visualization module of ABAQUS uses this output database for postprocessing results. ABAQUS “odbreport” utility creates organized reports of output database information in text, HTML, or CSV format. A script was written using this "odbreport" command to output temperature data for each output step in ASCII format. The thermal analysis output database file was used for this purpose. Another script was written to output the finite element mesh information including nodal coordinates and element connectivities.

For the structural analysis, a script was written to output nodal displacement data, stress tensor data, and the stress invariant data in ASCII format. The visualization data output are quite large especially because they are written in ASCII format. In the future, binary file containing suitable format would be provided.

\section{Immersive Visualizations}

\subsection{Introduction}

Immersive visualization simulates a true 3D environment. This means it can function as a virtual laboratory where measurement and analysis can be used to study the impact of fire on structures [6][7][8].

Smokeview is an OpenGL desktop visualization program that is currently used to visualize the results of an FDS simulation. It does not have the capability to display data in an IVE. The visualization methods developed for this project do have this capability. They can be displayed on a Linux desktop. Two approaches to the visualization of fire are described below: polygonal and volumetric.

Immersive visualization requires rendering with graphics hardware to achieve the 30 to 60 frame per second update rate necessary to provide the illusion that the user is truly immersed in the data. Graphics hardware are optimized for rendering of solid objects with polygonal models. The surfaces of the objects are represented by polygonal facets, and current graphics hardware can render millions of such polygons per second. Since the fire simulation is not a simple solid object, more creative methods are necessary. Two 
approaches were used. The first represents values in the simulation region as a collection of polygons, much as tracers in a 3-D flow. The second uses the computational power of the graphics processors to perform a volumetric 3D rendering within a shell geometry around the simulated volume. Both of these approaches are described below.

\subsection{Polygon Based Visualization of Fire}

After a FDS analysis, geometry and mesh data (3 dimensional array of points) along with the heat release rate data (as output from FDS analysis) were used for polygon based visualization of fire. Fire was represented by the heat release rate per unit volume (HRRPUV) and displayed with values ranging from 0 to 255. The initial goal for visualizing fire in the IVE was realism while emulating Smokeview's value cutoff approach. The cutoff was preserved in its original form; the project will not draw any fire with a lower HRRPUV value than the cutoff. The following polygon based volumetric algorithm was developed. Each cube in the mesh was subdivided according to a level of detail coefficient, and the HRRPUV values of interior points were interpolated linearly. For each of the resultant cubes, a random vertex was selected, and a tetrahedron was drawn between that point and the three adjacent points. The color and transparency of these tetrahedra were related to the HRRPUV value of the points. The visualization can be seen in Figure 5. A flow chart for the visualization is shown in Figure 6. It describes an algorithm used to create individual polygons in the fire. The algorithm uses inputs for the level of detail, density, and intensity to control if polygons are generated, where they are generated, and how they are colored. Several additional randomizations and features were added to the fire algorithm. First, the subdivision of any one cell was linearly randomized between subdivisions and the given level of detail, to give a more realistic effect. A density coefficient was also added to determine the chance a tetrahedron is drawn for a given cell. Finally, there is a cutoff for a minimum transparency for a tetrahedron, below which no polygons are drawn, to improve performance. The result was viewable in real time in the IVE.

The beam was visualized in two different ways. In one method, each frame of the beam was created individually, with data values for deformation, stress or temperature mapped to a color gradient. The ABAQUS files were used to find a boundary for the beam including displacement vectors, and only the shell was drawn. The disadvantage of this method is that the user cannot scale the offset, and sometimes the offset is very difficult to see. The solution is to allow dynamic scaling of the beam. This was accomplished using separate, rectangular prism elements translated and rotated to fit a specific element in ABAQUS's 3-D finite element model. The deflection values can be scaled by a user application, and rotation values can also be scaled at the same time to provide a smooth surface to the user. See Figure 7.

In addition to the beam visualization, a flow visualization was created. The user selects a point and the program numerically integrates the fluid flow velocities for a certain distance, and displays the generated curve. This limits the information being displayed to 
what the user is interested in, rather than displaying every single flow vector (see Figure 8).

\subsection{GPU Based Ray Traced Volumetric Visualization of Fire}

The second method to visualize the fire uses a graphics processing unit (GPU), but rather than using it for traditional polygon rendering, its parallel processing ability was used to trace rays through the volumetric data, computing the accumulated color and opacity of each ray using an emission/absorption model of light propagation through the simulated volume. According to this model, each point in the volume may emit light and also absorb light passing through it.

Like the polygon based visualization, this visualization method starts with the HRRPUV data from the FDS simulation for every computational step. These data are provided in a regular hexahedral 3D grid, and stored directly on the GPU in that form. In addition, the visualization uses a transfer function, mapping each HRRPUV value to a specific color and opacity per unit volume. In the emission/absorption model, the total opacity, $\alpha_{r}(t)$, was computed by integrating along the ray, where $s$ is the distance along the ray from the beginning at $s=0$ to $s=t$ :

$$
\alpha_{r}(t)=1-e^{-\int_{0}^{t} \kappa(\vec{r}(s)) d s}
$$

In this equation, $r(s)$ is the 3D ray position along the ray, and $\kappa$ is the absorption coefficient at each point in space. The color, $C_{r}(t)$, was computed by integrating the emitted light from $u=0$ to $u=t$ along the ray, accounting for the absorption through the volume to each point where the light was emitted:

$$
C_{r}(t)=\int_{0}^{t} C(\vec{r}(s)) e^{-\int_{0}^{s} \kappa(\vec{r}(u)) d u} d s
$$

For rendering, each of these integrals was evaluated numerically using a simple Euler integration, summing in uniform steps along the ray represented by the red arrows as shown in the left side of Figure 9. The transfer function was pre-processed by the method of Engel et al. [9] into a preintegrated 2D transfer table. For a unit integration step through the volume, the table provides a fast answer to the total color and opacity accumulated given the HRRPUV values at the start and end of the step. A sample of such a table can be seen in the lower square in Figure 9. The resulting image can be seen in the right side of Figure 9.

To allow non-unit steps through the volume, and accurate rendering of volume/object interactions, which can result in a partial step in the integration, a new approach was needed to scale the opacity and color from the preintegration table to a differing optical 
depth. Even with the same start and end values, if the ray travels farther, the absorption will be greater, and if it travels a shorter distance, the absorption will be less. If the volume emission and absorption are assumed to be piecewise constant over the step, a unit-step absorption of $\alpha$ will become $\alpha_{f}$ over a step of $f(f=\Delta(t))$ :

$$
\alpha_{f}=1-(1-\alpha)^{f}
$$

Similarly, if the emitted color within that unit step, modulated by the local absorption is $C$, the emitted color modulated by absorption for a step of $f$ will be $C_{f}$ :

$$
C_{f}=C \frac{\alpha_{f}}{\alpha}
$$

The limit of Equation (4) as $\alpha$ approaches 0 , indicating no absorption during the step, is

$$
C_{f}=C f
$$

Note that Engel et al. [9] present Equation (5) as the total partial-step scaling equation. While this is accurate for mostly transparent media, if there is any significant absorption, it does not give the correct result. This is particularly noticeable in fire visualization, as the fire burning on a wall or furniture produces exactly the situation where the approximation in (5) is least correct. This results in stair-step artifacts along the interface between the fire visualization and the solid wall or object. Using Equation (4) for the partial step integration in these circumstances resolves the problem and produces smooth and accurate visual results (see Figure 9).

These equations describe the integration along a single ray. To accelerate the rendering, parallel processors within the graphics processing unit were used to perform this ray integration for many rays simultaneously (see Figure 10). First any solid objects were rendered. These are used in the final display, but also allowed collection of a per-pixel maximum depth for each pixel to use in the ray integration. Second, the bounding box of the simulation volume was rendered. For each visible pixel of this box, the ray was integrated through that pixel until it reached the back of the box or one of the previously rendered solid objects. To allow the viewer to enter the volume, or slice away parts of it for better visibility, additional polygons were rendered where these clipping planes intersected the simulation bounding volume. Pixels on these polygons also use the ray integration to compute the final color.

This results in a high-quality interactive visualization for interactive exploration, either on the desktop or in the IVR. See Figure 11. 


\section{Discussion and Future Work}

In this initial study, three simulators were successfully linked: fire, thermal analysis of structures, and deformation of structures due to fire. The outputs from these three simulators were used in two visualization methods that were developed to display the results in an IVE. These plots can also be displayed in a desktop environment.

In the future, more complex simulations involving several structural members and connections will be performed. Typical office furniture along with different wall materials and thicknesses and ventilation would be used to conduct fire simulation. Furthermore, the visualizations in IVE would be enhanced with interactive measurement and analysis techniques and realistic depiction of charring of furniture due to fire. Some of the improvements planned are:

1. Geometric changes during visualization (ventilation, deformation)

2. Definition of cutting planes (orthogonal/arbitrary) / control volume

3. Extraction and proper display of numerical data at a specific location

4. Plot of multiple quantities with ability to turn on/off geometric features/outputs (e.g., smoke or SFRM)

5. Desktop environment with display of 3-D results and planar/tabular data in separate monitors/windows

6. Implementation of realistic visualization of window breakage

7. Implementation of smoke generation similar to those available in gaming software

In addition, current visualization capabilities will be enhanced. For example, in the polygon based visualization of fire the display of the beam as shown in Figure 7 will be refined such that no jagged edges develop while choosing a reasonable scaling factor for display.

\section{Acknowledgments}

Thanks are given to Kevin McGrattan and Glenn Forney for assistance with FDS/Smokeview.

\section{Disclaimer}

Certain commercial products may be identified in this paper in order to adequately describe the subject matter of this work. Such identification is not intended to imply recommendation or endorsement by the National Institute of Standards and Technology, nor is it intended to imply that the identified products are necessarily the best available for the purpose. 


\section{Bibliography}

1. McGrattan, K.B. (Ed.). 2004. Fire Dynamics Simulator (Version 4), Technical Reference Guide, NIST Special Publication 1018, National Institute of Standards and technology, Gaithersburg, Maryland, July, Retrieved from National Institute of Standards and Technology Building and Fire Research Lab Web site: http://fire.nist.gov/bfrlpubs/ fire07/PDF/f07053.pdf

2. Dassault Syestemes. 2008. ABAQUS Version 6.8, User’s manual. http://www.simulia.com

3. NIST (National Institute of Standards and Technology). 2005. Federal Building and Fire Safety Investigation of the World Trade Center Disaster: Final Report on the Collapse of the World Trade Center Towers. NIST NCSTAR 1. Gaithersburg, MD, September, p. 211.

4. Forney, G. P. (2008, July). User's Guide for Smokeview Version 5 - A Tool for Visualizing Fire Dynamics Simulation Data (NIST Special Publication No. 1017-1). Retrieved from National Institute of Standards and Technology Building and Fire Research Lab Web site: http://fds-smv.googlecode.com/svn/ trunk/FDS/trunk/Manuals/All_PDF_Files/SMV_5_User_Guide.pdf

5. Chen, W. and Deng, X. (2000). Performance of Shell Elements in Modeling Spotwelded Joints. Finite Elements in Analysis and Design, 35(1), pp. 41-57.

6. Hagedorn, J. G., Terrill, J. E., Peskin, A. P., \& Filliben, J. J. (n.d.). Methods for Quantifying and Characterizing Errors in Pixel Based 3D Rendering. Retrieved June 3, 2008, from http://math.nist.gov/mcsd/savg/vis/metrology/visMet1.pdf

7. Hagedorn, J. G., Dunkers, J., Satterfield, S., Peskin, A. P., Kelso, J. T., \& Terrill, J. E. (2007, September-October). Measurement Tools for the Immersive Visualization Environment: Steps Toward the Virtual Laboratory, NIST Journal of Research, 112 (5), pp.257-270, from http://nvl.nist.gov/pub/nistpubs/jres/112/5/V112.N05.A02.pdf

8. Terrill, J. E., Satterfield, S., Hagedorn, J. G., Kelso, J. T., Peskin, A. P., George, W. L., Griffin, T. J., Hung, H. K., and Kriz, R. D. (2005). Science at the Speed of Thought, Ambient Intelligence for Scientific Discovery: Lecture Notes in Artificial Intelligence, 3345, pp. 1-24, from http://math.nist.gov/mcsd/savg/papers/VLab-AIpaper.pdf

9. Engel, K., Hadwiger, M., Kniss, J. M., Rezk-Salama, C. and Weiskopf, D. (2006). Real-Time Volume Graphics, A K Peters, Wellesey, MA. 


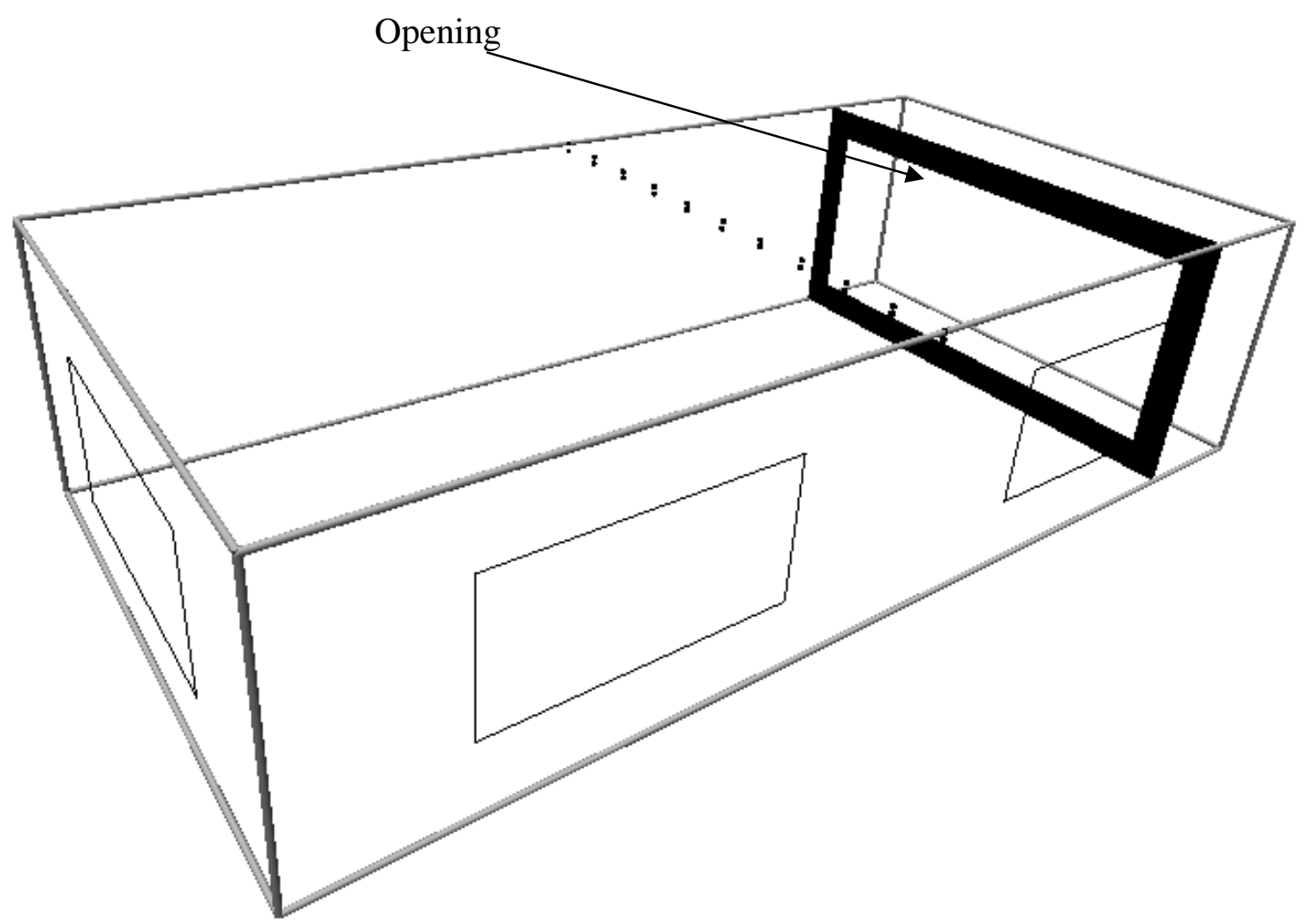

Figure 1: Building structure used as test case. Points across the top represent locations where data is collected for subsequent thermal and structural analyses 


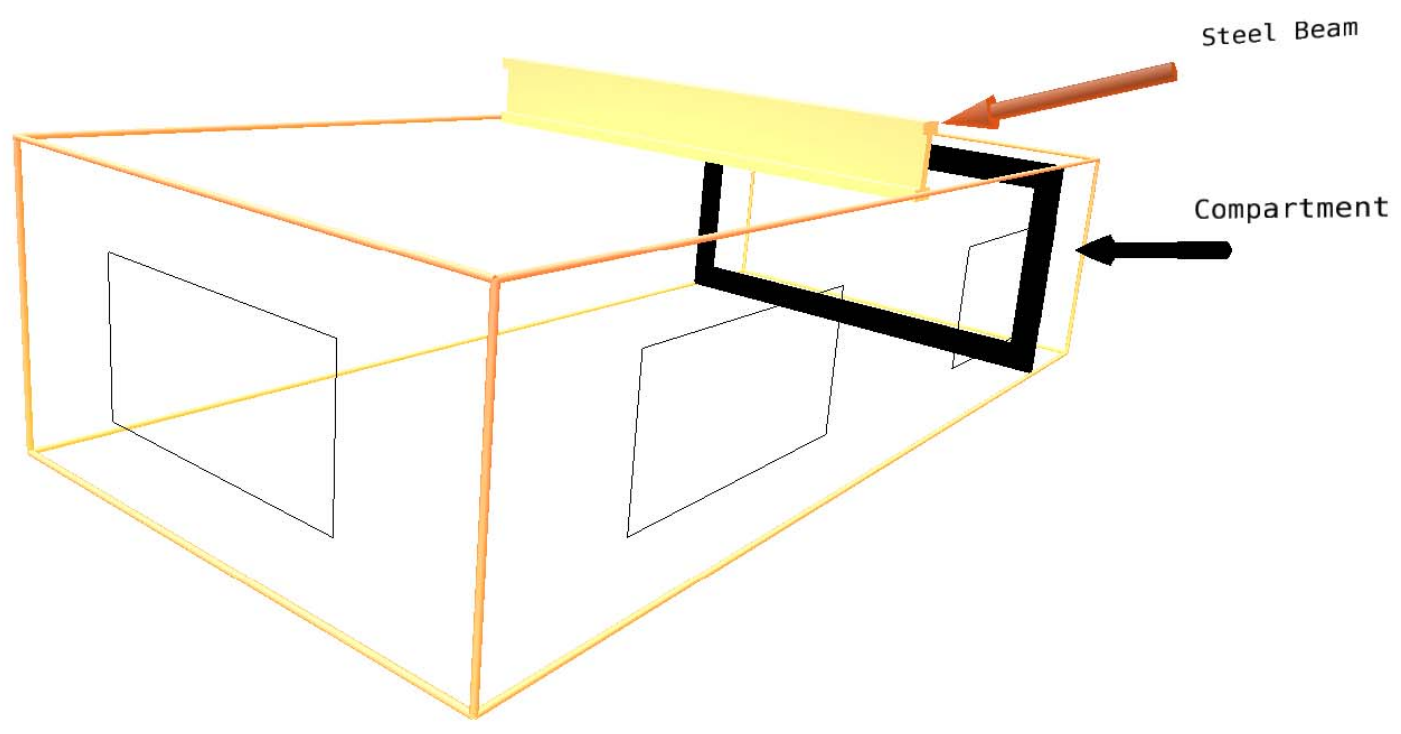

Figure 2: Location of the beam in room 


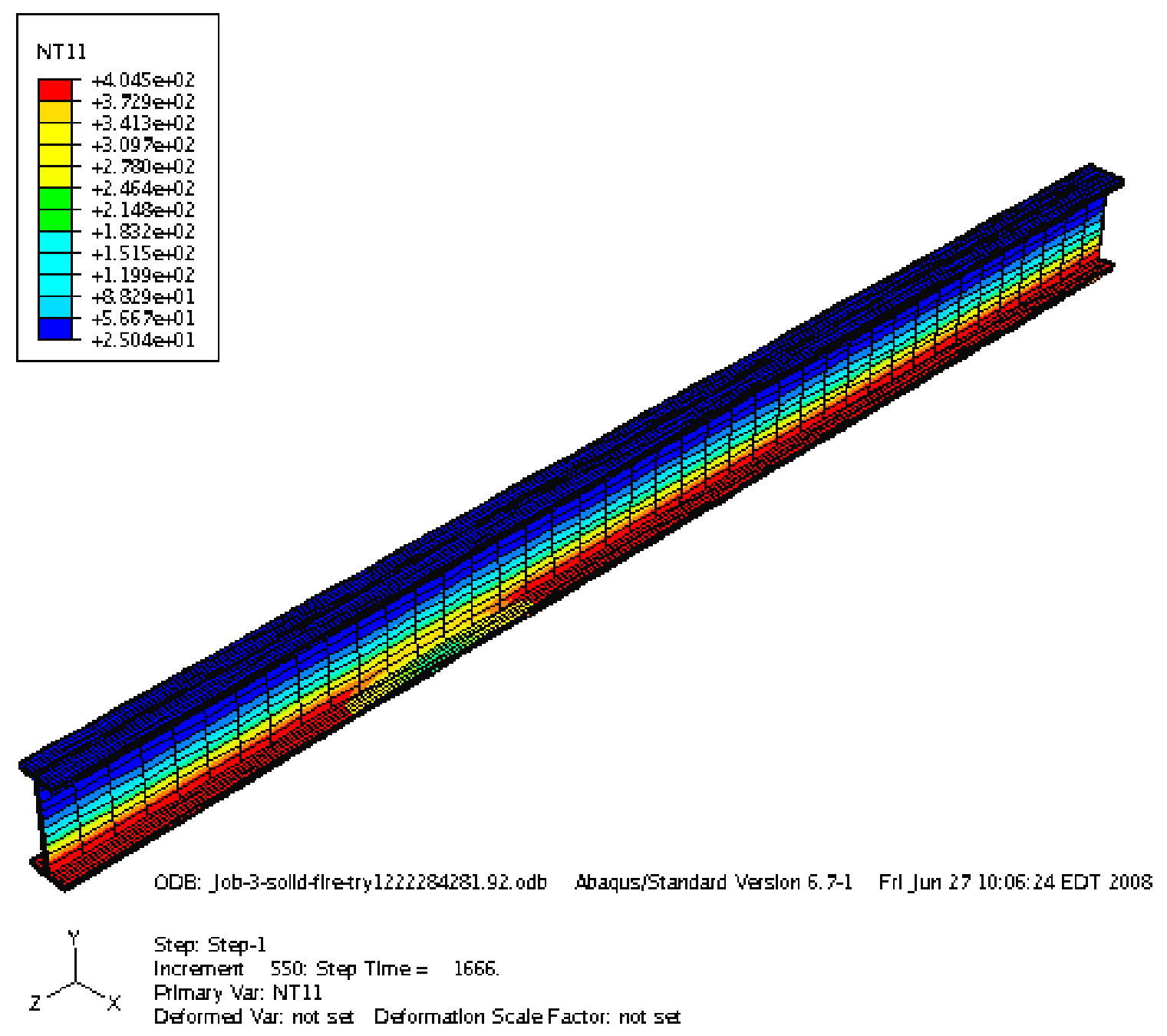

Figure 3: Temperature profile in the beam after 1666 seconds as computed by the ABAQUS thermal model using 3-D element. 


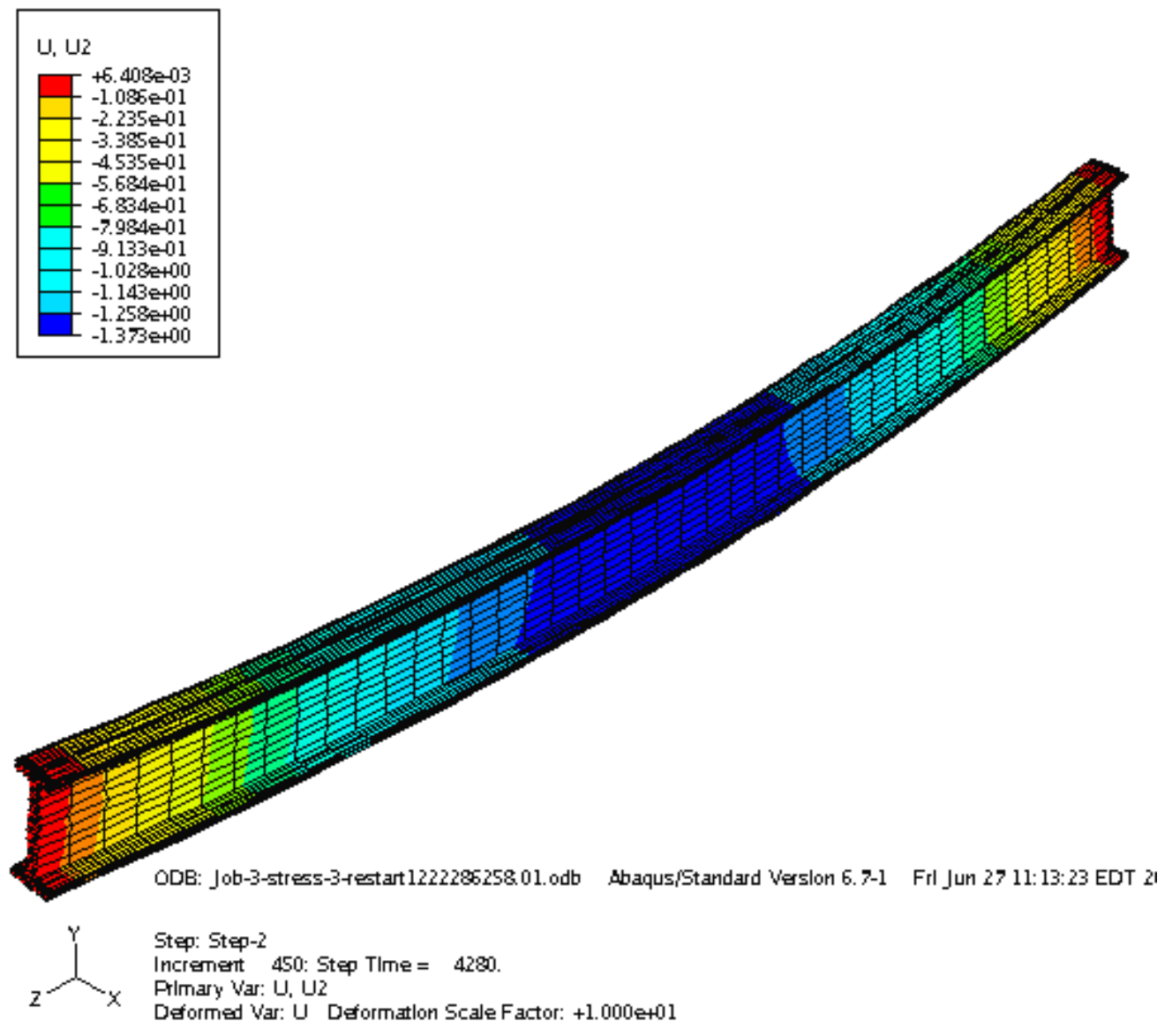

Figure 4: Vertical displacement in the beam as computed using the sequential thermalstructural analyses in ABAQUS. 


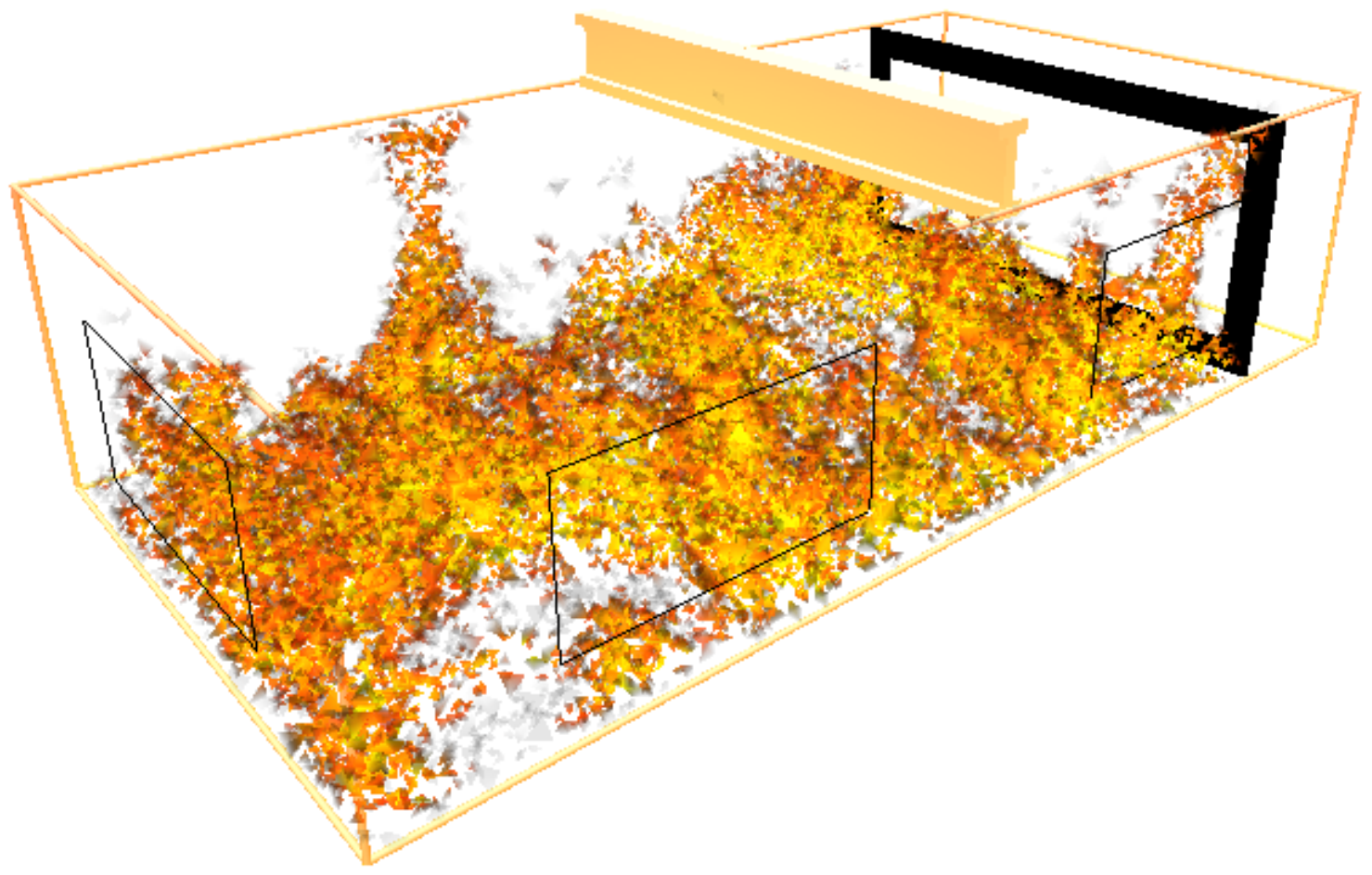

Figure 5: Polygon based fire visualization that is displayable in the IVE. 


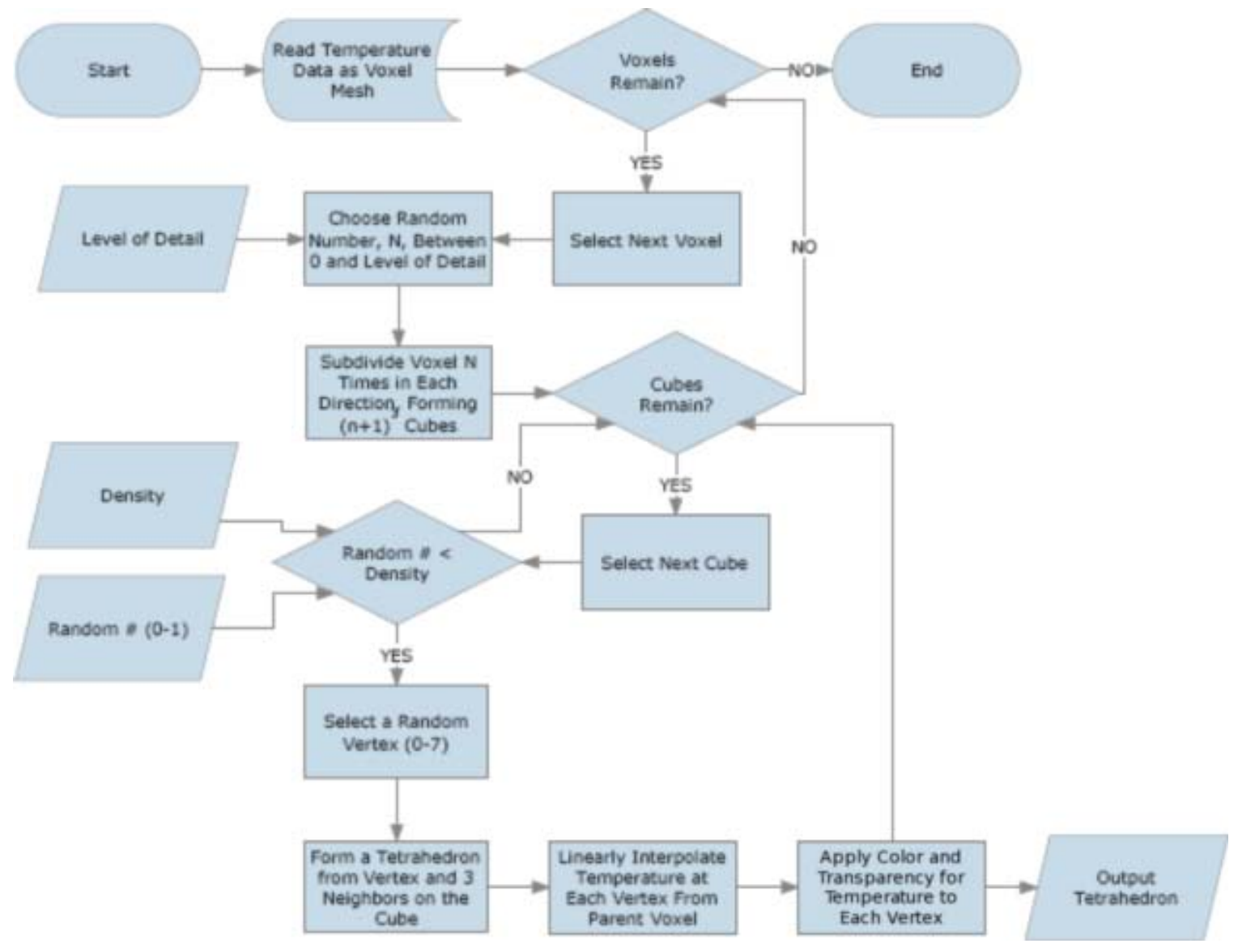

Figure 6. A flowchart for polygon based fire visualization. 


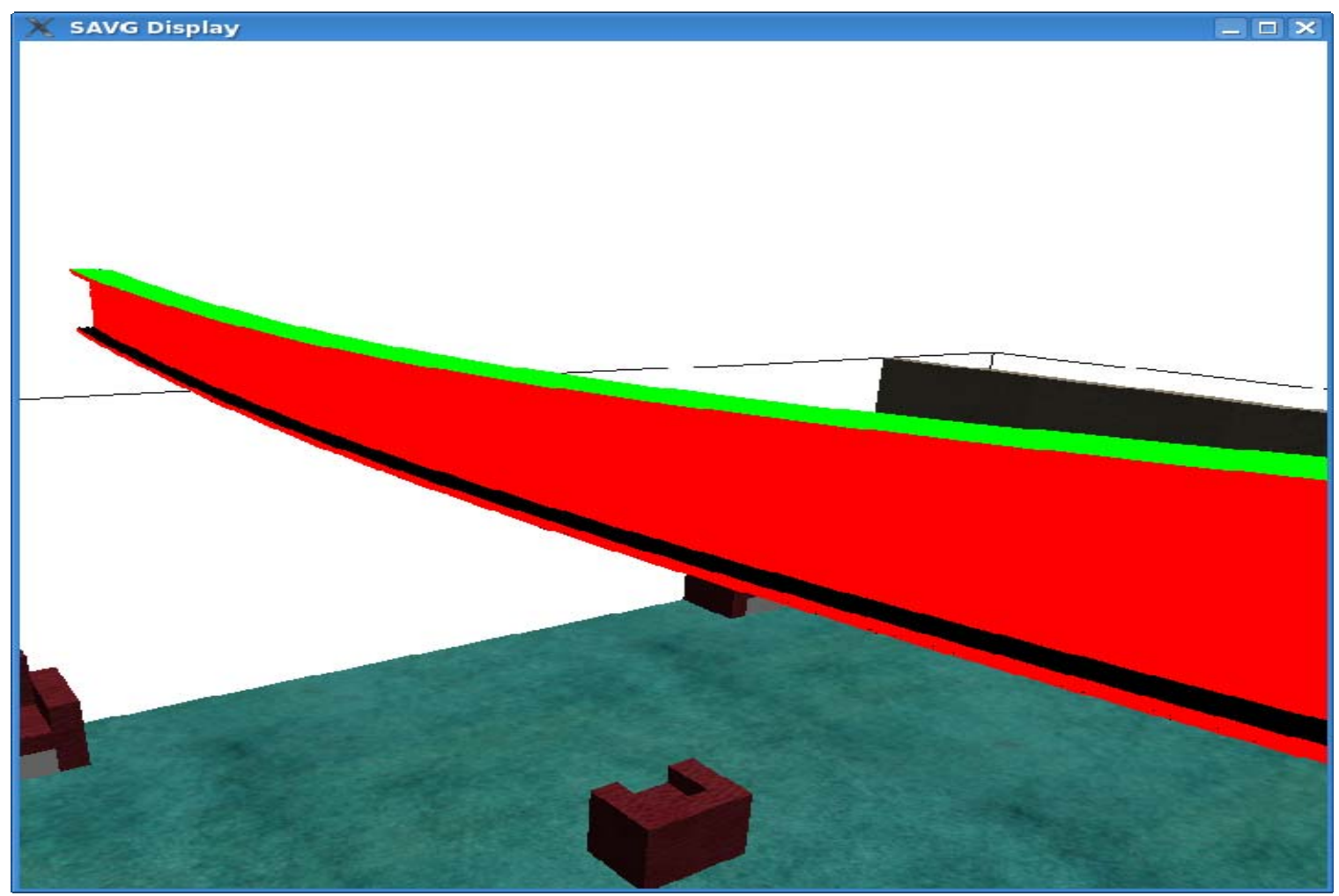

Figure 7: Beam deformation due to fire. 


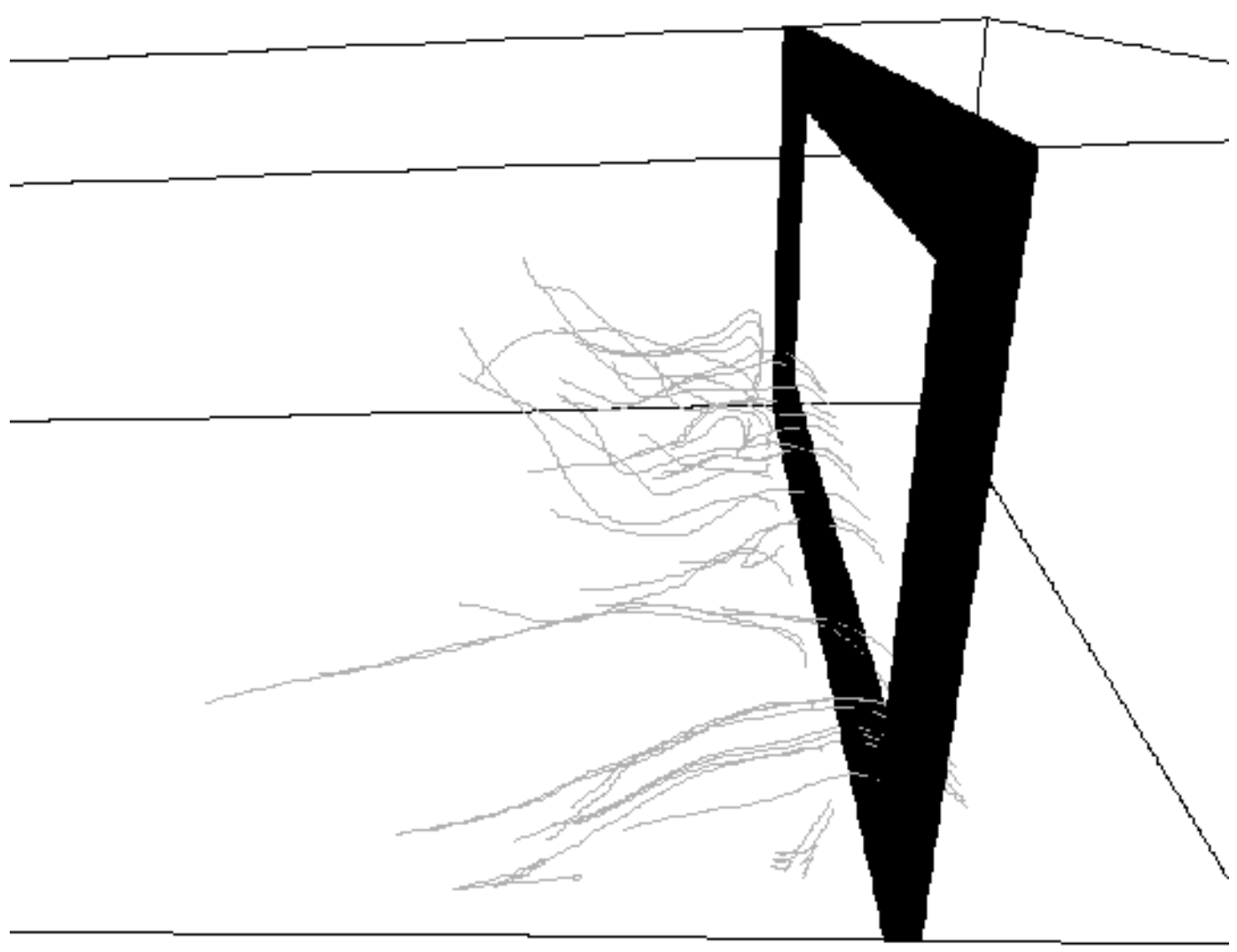

Figure 8: User selected flow streams. 


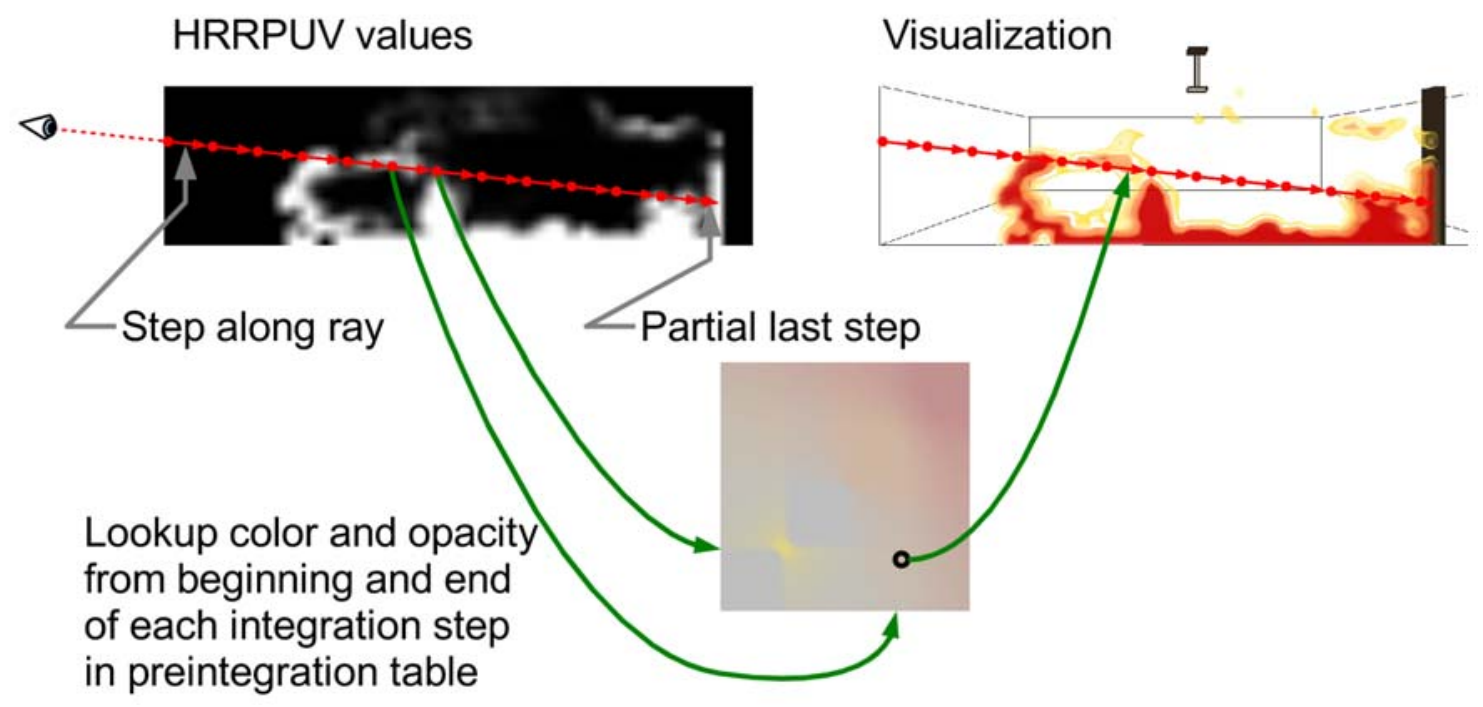

Figure 9: Volume integration along a ray. 


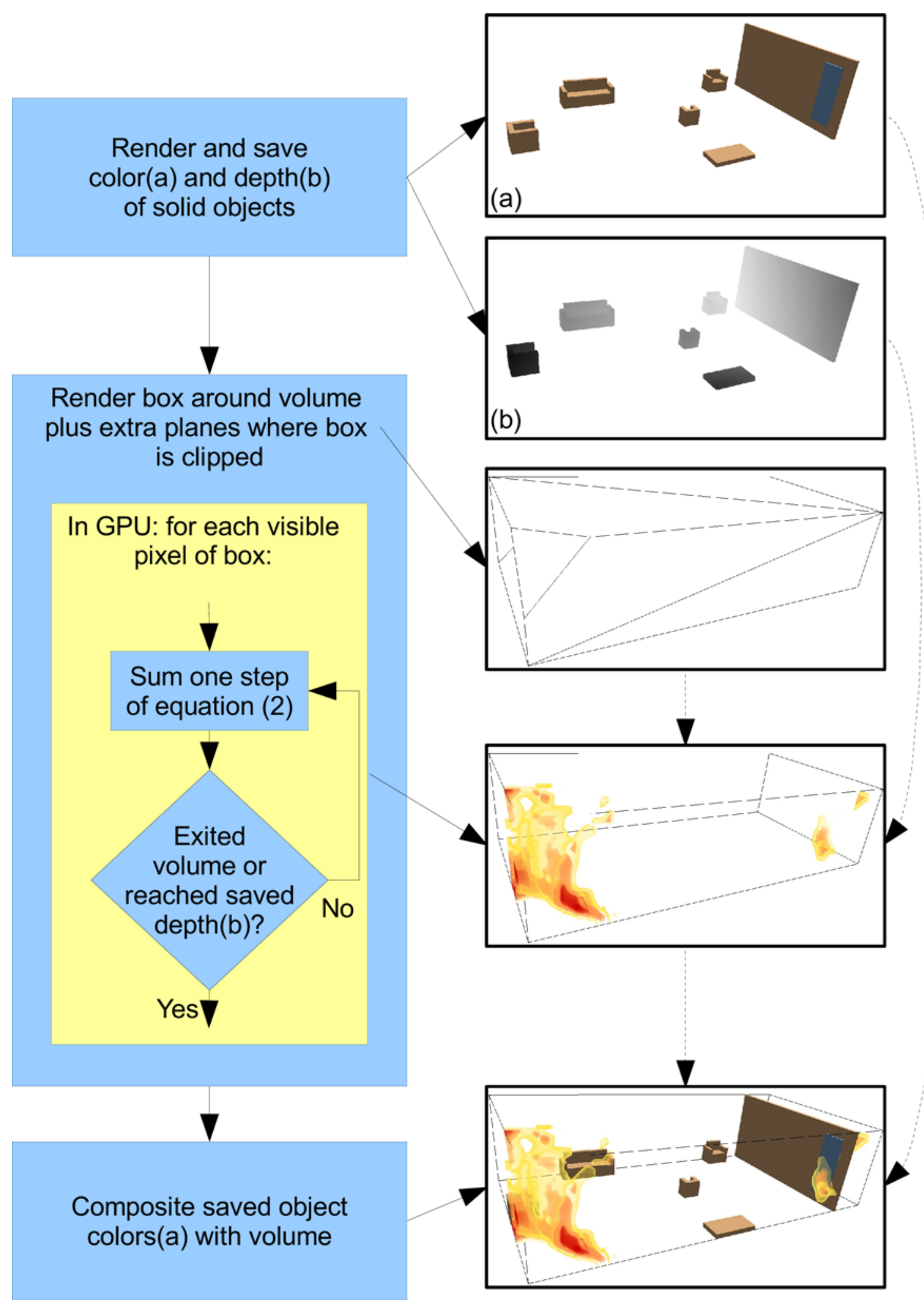

Figure 10: Flowchart of GPU-based volumetric fire rendering. 


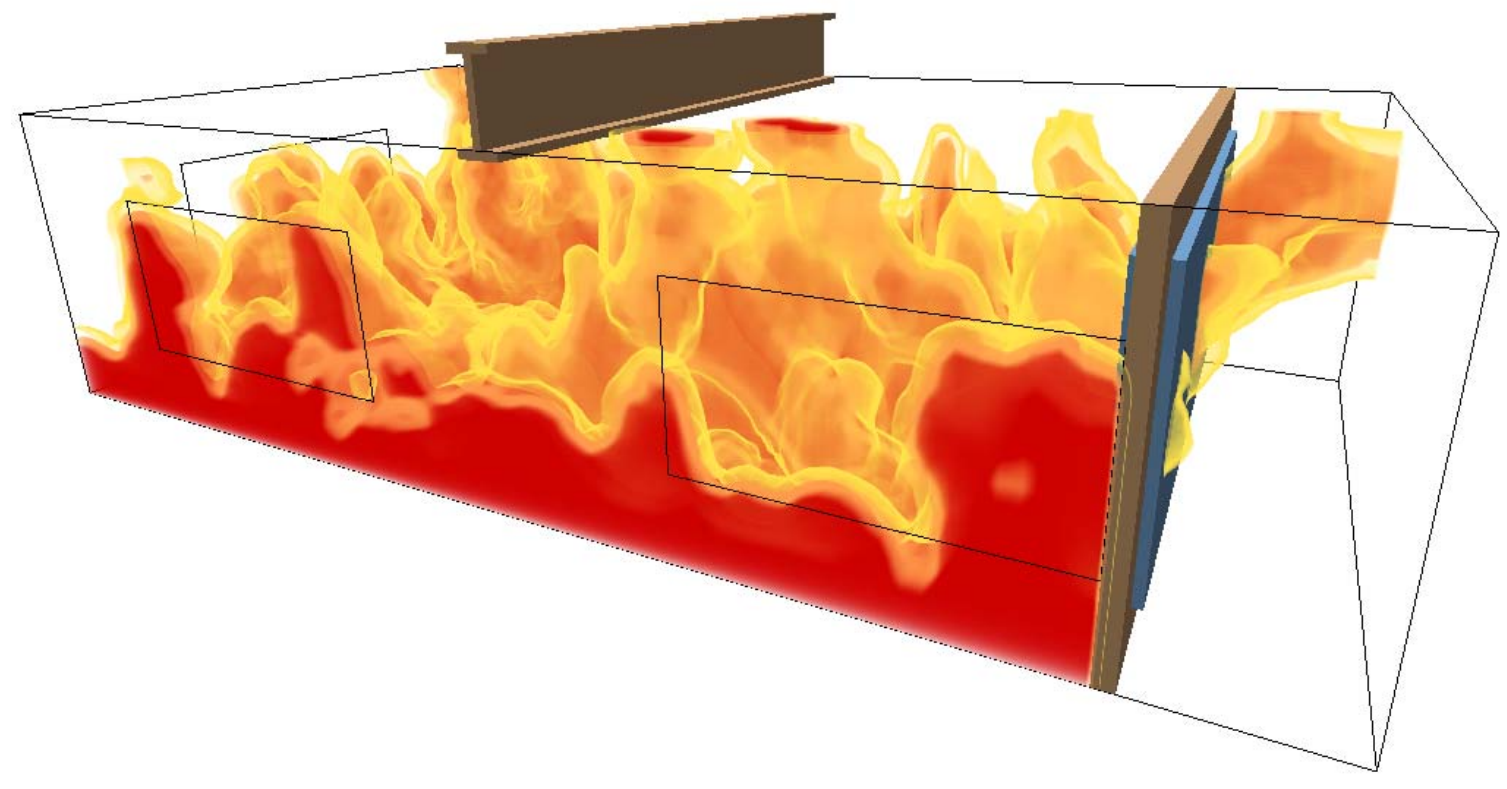

Figure 11: GPU based ray traced volumetric rendering of fire. 


\section{Appendix 1: FDS Input File for the Test case}

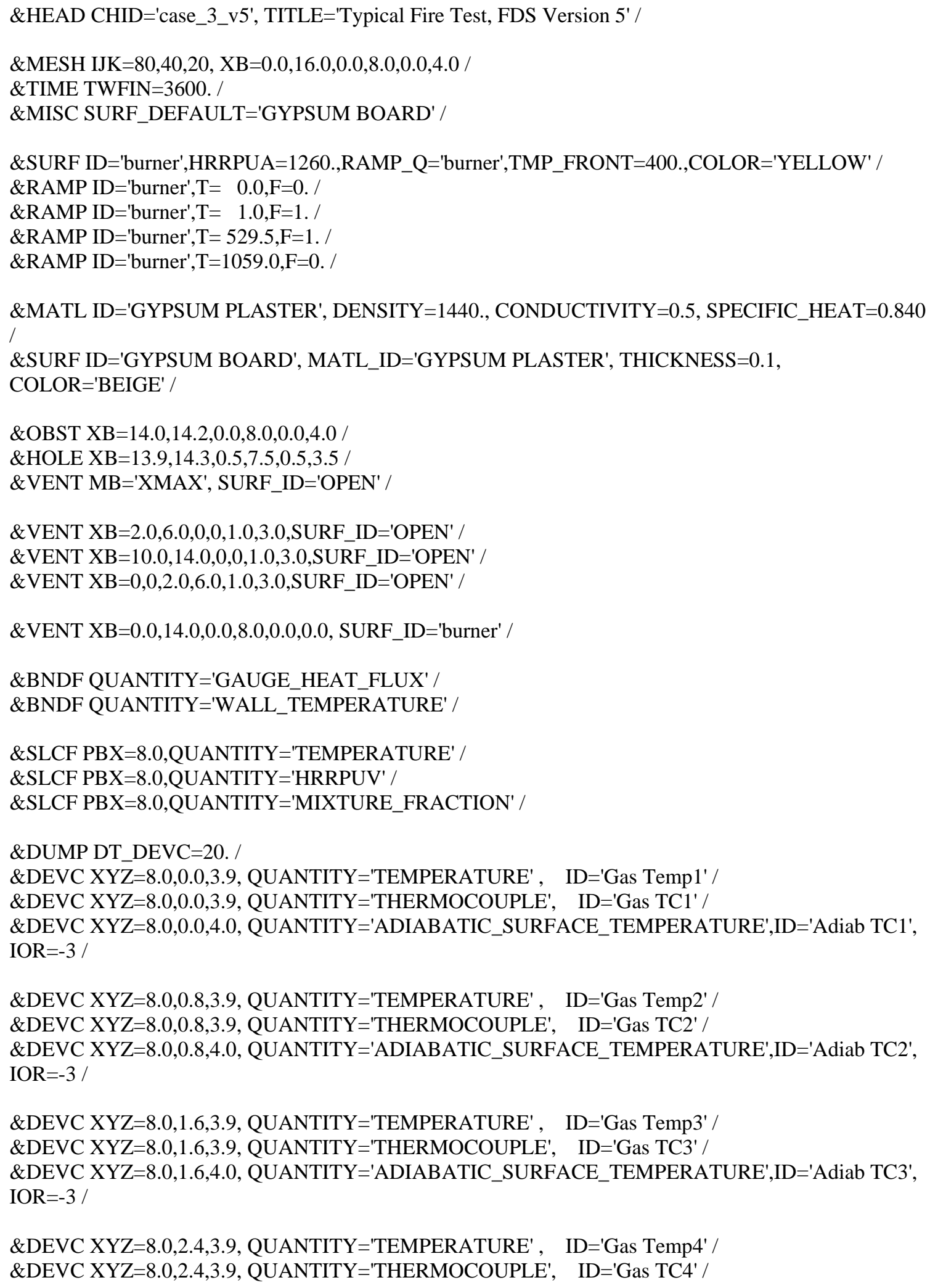


\&DEVC XYZ=8.0,2.4,4.0, QUANTITY='ADIABATIC_SURFACE_TEMPERATURE',ID='Adiab TC4', $\mathrm{IOR}=-3 /$

\&DEVC XYZ=8.0,3.2,3.9, QUANTITY='TEMPERATURE' , ID='Gas Temp5' /

\&DEVC XYZ=8.0,3.2,3.9, QUANTITY='THERMOCOUPLE', ID='Gas TC5' /

\&DEVC XYZ=8.0,3.2,4.0, QUANTITY='ADIABATIC_SURFACE_TEMPERATURE',ID='Adiab TC5', $\mathrm{IOR}=-3 /$

\&DEVC XYZ=8.0,4.0,3.9, QUANTITY='TEMPERATURE' , ID='Gas Temp6' /

\&DEVC XYZ=8.0,4.0,3.9, QUANTITY='THERMOCOUPLE', ID='Gas TC6' /

\&DEVC XYZ=8.0,4.0,4.0, QUANTITY='ADIABATIC_SURFACE_TEMPERATURE',ID='Adiab TC6', $\mathrm{IOR}=-3 /$

\&DEVC XYZ=8.0,4.8,3.9, QUANTITY='TEMPERATURE' , ID='Gas Temp7' /

\&DEVC XYZ=8.0,4.8,3.9, QUANTITY='THERMOCOUPLE', ID='Gas TC7' /

\&DEVC XYZ=8.0,4.8,4.0, QUANTITY='ADIABATIC_SURFACE_TEMPERATURE',ID='Adiab TC7', $\mathrm{IOR}=-3 /$

\&DEVC XYZ=8.0,5.6,3.9, QUANTITY='TEMPERATURE' , ID='Gas Temp8' /

\&DEVC XYZ=8.0,5.6,3.9, QUANTITY='THERMOCOUPLE', ID='Gas TC8' /

\&DEVC XYZ=8.0,5.6,4.0, QUANTITY='ADIABATIC_SURFACE_TEMPERATURE',ID='Adiab TC8', $\mathrm{IOR}=-3 /$

\&DEVC XYZ=8.0,6.4,3.9, QUANTITY='TEMPERATURE' , ID='Gas Temp9' / \&DEVC XYZ=8.0,6.4,3.9, QUANTITY='THERMOCOUPLE', ID='Gas TC9' /

\&DEVC XYZ=8.0,6.4,4.0, QUANTITY='ADIABATIC_SURFACE_TEMPERATURE',ID='Adiab TC9', IOR=-3 /

\&DEVC XYZ=8.0,7.2,3.9, QUANTITY='TEMPERATURE' , ID='Gas Temp10' /

\&DEVC XYZ=8.0,7.2,3.9, QUANTITY='THERMOCOUPLE', ID='Gas TC10' /

\&DEVC XYZ=8.0,7.2,4.0, QUANTITY='ADIABATIC_SURFACE_TEMPERATURE',ID='Adiab TC10', $\mathrm{IOR}=-3 /$

\&DEVC XYZ=8.0,7.95,3.9, QUANTITY='TEMPERATURE' , ID='Gas Temp11' /

\&DEVC XYZ=8.0,7.95,3.9, QUANTITY='THERMOCOUPLE', ID='Gas TC11' /

\&DEVC XYZ=8.0,7.95,4.0, QUANTITY='ADIABATIC_SURFACE_TEMPERATURE',ID='Adiab

TC11', IOR=-3 /

\&TAIL / 


\section{List of Figures}

Figure 1: Building structure used as test case. Points across the top represent locations where data is collected for subsequent thermal and structural analyses

Figure 2: Location of the beam in room

Figure 3: Temperature profile in the beam after $1666 \mathrm{~s}$ as computed by the ABAQUS thermal model using 3-D element.

Figure 4: Vertical displacement in the beam as computed using the sequential thermalstructural analyses in ABAQUS.

Figure 5: Polygon based fire visualization that is displayable in the IVE.

Figure 6: A flowchart for polygon based fire visualization.

Figure 7: Beam deformation due to fire.

Figure 8: User selected flow streams.

Figure 9: Volume integration along a ray.

Figure 10: Flowchart of GPU-based volumetric fire rendering.

Figure 11: GPU based ray traced volumetric rendering of fire. 